\title{
Efficacy and Safety of Teneligliptin in Patients of Type 2 Diabetes Mellitus with Chronic Kidney Disease: ATEND-CKD Study
}

\author{
Dr. Mayuresh Dilip Kiran ${ }^{* 1}$, Dr. Monali Vakharia ${ }^{2}$, Lalit Jeevan Pawaskar ${ }^{3}$, Shaheen Naseem Sheikh ${ }^{4}$ \\ ${ }^{1}$ Vice President, Medical Services, ${ }^{2}$ Manager, Medical Services, ${ }^{3}$ Executive, Pharmacovigilance, \\ ${ }^{4}$ Research Associate, Pharmacovigilance, \\ Centaur Pharmaceuticals Pvt. Ltd
}

\section{Corresponding Author -}

Dr. Mayuresh Kiran

Centaur Pharmaceuticals Pvt. Ltd.,

Centaur House, Near Grand Hyatt, Vakola, Santacruz (East), Mumbai - 400055

Email: mayuresh_kiran@rediffmail.com

\begin{abstract}
Background: Prior studies have estimated prevalence of CKD among non-institutionalized adults with type 2 diabetes mellitus (T2DM) at 34.5$42.3 \%$, with most CKD cases identified as early stage (stage 1 or 2). Diabetes affects many organs, and complications due to high blood glucose are an important cause of disability, reduced quality of life, and premature death. (8) There is paucity of data regarding efficacy and safety of teneligliptin in T2DM with chronic kidney disease (CKD) especially in Indian population and therefore this study was planned. Methods: This was a phase IV, multi-centric, open labelled, non-comparative, user initiated study done in 405 patients diagnosed with CKD due to uncontrolled T2DM. Patients were given samples of Teneligliptin $20 \mathrm{mg}$ at initial visit at day 1 and asked to take one tablet daily for a total of 90 days. Glycemic and renal profile during initial and follow up visit was used for efficacy evaluation. Patients were instructed to keep a diary to record daily symptoms and adverse events if any for safety evaluation. Results: Teneligliptin reported significant mean reduction in FPG and PPBG by 40.4 and $57.5 \mathrm{mg} / \mathrm{dl}$ respectively. At 12 weeks, statistically significant $0.9 \%$ reduction in HbA1c was noted. Effects on renal parameters were also found significantly positive in this study, with reduction of Sr. creatinine by around $4 \%$ and reduction in the BUN of around $9 \%$. There was decrease in mean urinary albumin and UACR and increase in mean eGFR, though it was not statistically significant. Among all the patients, $6.41 \%$ of patients experienced adverse event. Hypoglycemia was the most common adverse event seen in $4.44 \%$ of patients followed by constipation (1.23\%) and gastritis $(0.74 \%)$. Conclusion: The study reported significant improvement in glycaemic control, serum creatinine and BUN with teneligliptin.
\end{abstract}

Keywords: Teneligliptin, serum creatinine, BUN, chronic kidney disease, HbAlc

\section{Introduction}

India, experiencing swift socioeconomic progress and urbanization, carries a considerable share of the global diabetes burden. The prevalence of diabetes is increasing not only in urban populations, but also in rural populations as a result of the urbanization of lifestyle parameters along with high prevalence of prediabetes. ${ }^{[1]}$ According to Wild et al. ${ }^{[2]}$ the prevalence of diabetes is predicted to double globally from 171 million in 2000 to 366 million in 2030 with a maximum increase in India. It is predicted that by 2030 diabetes mellitus may afflict up to 79.4 million individuals in India, while China (42.3 million) and the United States (30.3 million) will also see significant increases in those affected by the disease. ${ }^{[3]}$

The glycemic control in treated patients is not ideal because of the considerable disparity in the availability and affordability of diabetes care, as well as low awareness of the disease. This may increase the occurrence of vascular complications. ${ }^{[1]}$ Diabetes mellitus is the most common contributor to chronic kidney disease (CKD) in the USA and worldwide. Prior studies have estimated prevalence of CKD among non-institutionalized adults with type 2 diabetes mellitus (T2DM) at $34.5-42.3 \%$, with most CKD cases identified as early stage (stage 1 or 2 ). Additionally, diabetes is present in approximately $30-40 \%$ of all cases of end-stage renal disease (ESRD) in the USA. Antidiabetes medication for concurrent T2DM with CKD involves use of one or more drugs among insulin, biguanides, sulfonylureas, thiazolidinediones and dipeptidyl peptidase-4 inhibitors. ${ }^{[4]}$

Dipeptidyl peptidase-4 inhibitors (DPP-4Is) and glucagon-like peptide-1 receptor agonists (GLP-1RAs) have been the important part of therapy for type 2 diabetes mellitus (T2DM) since the evolution of incretin science. DPP-4Is are particularly popular as they are oral drugs, less costly than GLP-RAs, with modest to 


\section{International Journal of Innovative Research in Medical Science (IJIRMS) Volume 04 Issue 01 Jan 2019, ISSN: 2455-8737, Imp. Factor - 4.102 \\ Available online at $-\underline{w w w . i j i r m s . i n}$}

moderate glucose lowering similar to sulfonylureas (SU) depending on the baseline glycemic load. ${ }^{[5]}$ They generally do not cause hypoglycemia and possess weight-neutral or weight losing properties. ${ }^{[6]}$ DPP-4Is inhibit the principal enzyme responsible for the degradation of endogenous GLP-1. By decreasing clearance of GLP-1, concentrations of active GLP-1 are increased, resulting in a lowering of fasting and postprandial glucose concentrations. They also improve $\beta$-cell responsiveness to prevailing glucose concentrations and suppress glucagon secretion. ${ }^{[7]}$

Teneligliptin is a recently developed oral DPP-4I indicated for the management of T2DM in adults along with diet and exercise. Teneligliptin has been recently available in Japan, Argentina, and India at relatively affordable price. This is a positive step toward the management of T2DM in developing countries, where the cost of medicine is out-of-pocket expenditure and is a limiting factor for health care ${ }^{[8]}$ Also, a study recently suggested that the glycemic response to incretin-based therapies could differ ethnicity-wise with Asians having better response to it. ${ }^{[6]}$

Teneligliptin has been evaluated in T2DM as monotherapy with diet and exercise and also in combination with metformin, glimepiride, pioglitazone, and insulin in short-term (12 weeks) and long-term (52 weeks) studies. These studies have reported a reduction in HbAlc of $0.8 \%-0.9 \%$ within 12 weeks of therapy. Two 52-week studies reported sustained improvement in glycemic control with teneligliptin. Diabetes affects many organs, and complications due to high blood glucose are an important cause of disability, reduced quality of life, and premature death. ${ }^{[8]}$ There is paucity of data regarding efficacy and safety of teneligliptin in T2DM with chronic kidney disease (CKD) especially in Indian population and therefore this study was planned for Assessing Teneligliptin in Diabetes with CKD (ATEND-CKD).

\section{Methodology}

This was a phase IV, multicentric, open labelled, non-comparative, user initiated study conducted in 20 Centers across India from Jan 2018- June 2018. A total of 419 patients were enrolled in the study with 14 patients lost to follow up and hence data of 405 patients was analyzed.

\section{Inclusion and Exclusion criteria}

Patients diagnosed with CKD due to uncontrolled T2DM (Hba1c between 7.0 to $10.0 \%$ with eGFR $<90 \mathrm{ml} / \mathrm{min}$ and $\geq 15 \mathrm{ml} / \mathrm{min}$ i.e. Stage 1 to 4 of CKD and FBS >126mg/dl and PPBS > 180mg/dl) and willing to participate in the study were enrolled. Patients of both the gender and $>18$ years of age were recruited for this study.

Patients with type 1 diabetes mellitus, elevated levels of hepatic enzymes, eGFR $<15 \mathrm{ml} / \mathrm{min}$ (Stage 5 of CKD, i.e. end stage renal disease), current or previous history of cancers, severe liver disease, history of gestational diabetes, hypersensitivity to teneligliptin and who cannot adhere to the protocol (mentally ill and patients with psychological problem) were excluded from the study. Patients with CKD due to causes other than diabetes were also excluded.

\section{Study procedure}

All eligible patients were informed about the nature of the study and written consent was taken to participate in the study. A detailed medical history was obtained from all enrolled patients, followed by thorough clinical examination. Patients were given free physician samples of TEN 20 (Teneligliptin $20 \mathrm{mg}$ ) at initial visit at day 1 and asked to take one tablet daily for a total of 90 days. Follow up visit was scheduled at day 90 for efficacy and safety assessment after initial assessment at day 1. Glycemic and renal profile during initial and follow up visit was used for efficacy evaluation. Patients were instructed to keep a diary to record daily symptoms and adverse events if any. In case of any safety-related issues and adverse events or serious adverse events, the investigator withdrew the patient from the trial and treated accordingly. Along with teneligliptin, other medications for CKD were continued.

\section{Efficacy and Safety assessment}

The primary efficacy assessment was done by analyzing the change in glycemic profile. Glycemic profile included fasting blood sugar (FBS), post-prandial blood sugar (PPBS) and HbA1c measurement.

The secondary efficacy assessment was done by analyzing the change in renal profile which included serum creatinine, blood urea nitrogen (BUN), eGFR, urinary albumin and urine albumin: creatinine ratio (UACR).

At follow up visit, patients were asked for any adverse events and their diaries were assessed. Adverse events were noted and classified into two categories as serious or non-serious. Adverse events observed were followed up at each visit and treated if necessary by the investigators till their resolution.

\section{Ethical and Regulatory matters}

The study drug is currently available in India and classified under schedule $\mathrm{H}$ drugs i.e. it can be sold only in the presence of a prescription of a registered medical practitioner. All the patients recruited in the study have read informed consent form and signed the same. Clinical trial protocol, ICF, CRF, undertaking by the investigators form, ethical committee certificates were collected before initiating the clinical study.

\section{Statistical analysis}

The data collected was entered into Microsoft excel 2016 and analyzed using mean and standard deviation. Efficacy endpoints before and after the treatment in patients were analysed by using paired t-test. A ' $p$ ' value $<0.05$ was considered statistically significant.

\section{Results}

A total of 419 patients were enrolled. The mean age of patients in our study was 56.4 years. Out of all patients, 290 were male and 129 were female. 14 patients were lost to follow up and so data of 405 patients was analyzed.

The mean FBS at baseline visit 1 was $159.8 \pm 32.47 \mathrm{mg} / \mathrm{dl}$ and at final visit 2 was $119.4 \pm 26.17 \mathrm{mg} / \mathrm{dl}$. Similarly, the mean PPBS at baseline visit 1 was $238.3 \pm 57.81 \mathrm{mg} / \mathrm{dl}$ and at final visit 2 was $180.8 \pm 44.22 \mathrm{mg} / \mathrm{dl}$. This decrease in mean FBS and PPBS was statically very significant i.e $\mathrm{p}<0.0001$ (Fig 1) 


\section{International Journal of Innovative Research in Medical Science (IJIRMS) Volume 04 Issue 01 Jan 2019, ISSN: 2455-8737, Imp. Factor - 4.102 \\ Available online at $-\underline{w w w . i j i r m s . i n}$}

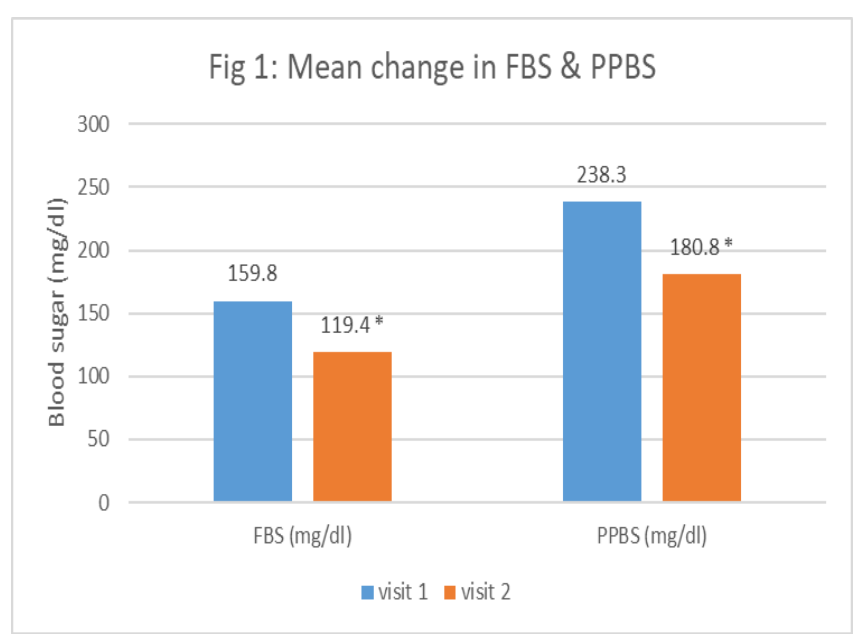

*Statistically significant

The decrease in mean $\mathrm{HbA} 1 \mathrm{c}$ from $7.8 \pm 1.1 \%$ at baseline to $6.9 \pm$ $1.3 \%$ at final visit was also statistically very significant. (p < 0.0001 ) (fig 2)

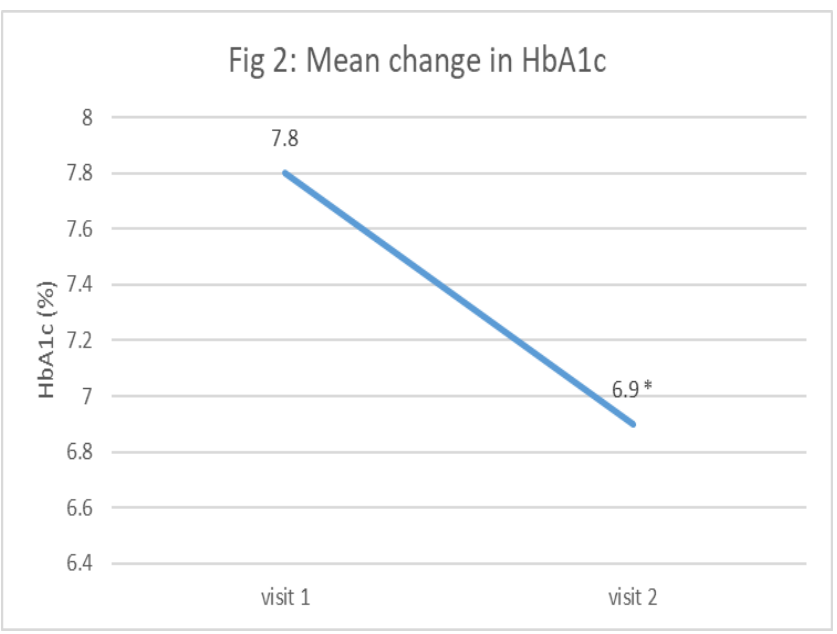

*Statistically significant

The mean serum creatinine value at baseline was $2.6 \pm 1.3 \mathrm{mg} / \mathrm{dl}$ and at final visit was $2.5 \pm 1.2 \mathrm{mg} / \mathrm{dl}$. This mean change was statistically significant. $(\mathrm{p}<0.0001)($ fig 3$)$
Fig 3: Mean change in serum creatinine

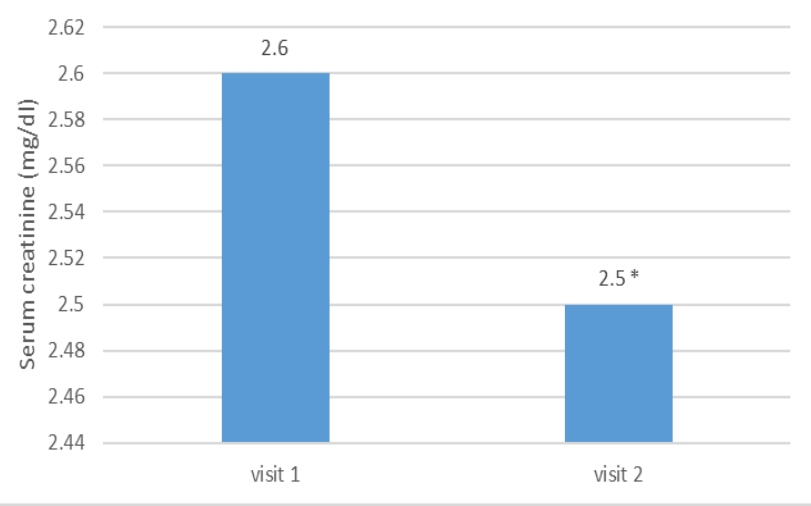

*Statistically significant

The mean BUN level at baseline was $40.3 \pm 19.97 \mathrm{mg} / \mathrm{dl}$ and at final visit was $36.7 \pm 17.65 \mathrm{mg} / \mathrm{dl}$. This decrease in mean BUN level was statistically significant $(\mathrm{p}<0.05)$. The mean change in eGFR from $43.2 \pm 25.9$ to $43.8 \pm 27.5 \mathrm{ml} / \mathrm{min} / 1.73 \mathrm{~m} 2$ was not statistically significant. (fig 4)

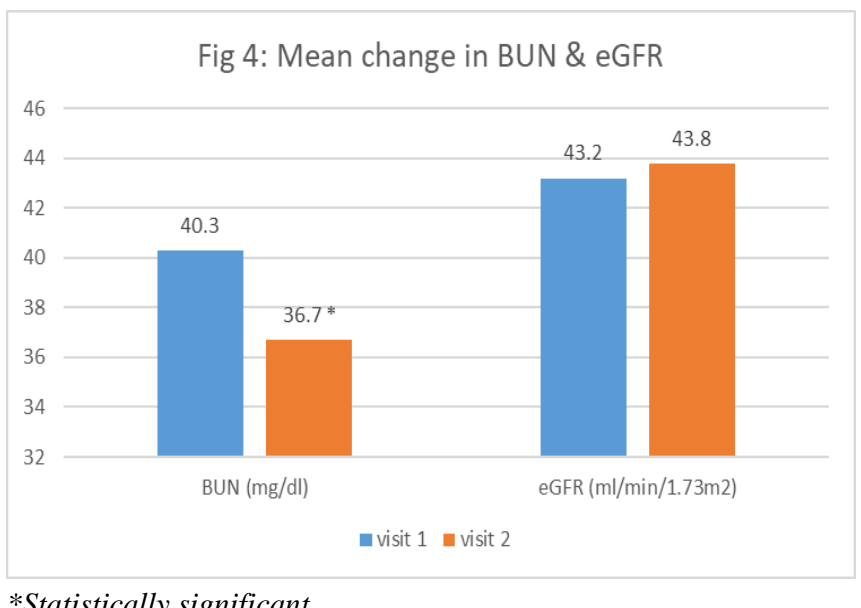

\section{*Statistically significant}

Also, there was decrease in mean urinary albumin levels from $268.3 \mathrm{mg} / \mathrm{dl}$ at baseline to $190.1 \mathrm{mg} / \mathrm{dl}$ at final visit but this decrease was not statistically significant. Similarly, the mean decrease in UACR from $160.1 \mathrm{mg} / \mathrm{g}$ at baseline to $152.9 \mathrm{mg} / \mathrm{g}$ at final visit was not statistically significant.

Table 1 shows mean reduction in values of all the parameters of glycemic and renal profile from baseline to final visit. This mean reduction was statistically significant for all the parameters except for urinary albumin, eGFR and UACR.

Table 1: Mean reduction in glycemic and renal profile compared to baseline

\begin{tabular}{|c|c|c|c|c|c|c|c|c|}
\hline & $\begin{array}{c}\text { FBS } \\
(\mathrm{mg} / \mathrm{dl})\end{array}$ & $\begin{array}{c}\text { PPBS } \\
(\mathrm{mg} / \mathrm{dl})\end{array}$ & $\begin{array}{c}\text { HbA1c } \\
(\%)\end{array}$ & $\begin{array}{c}\text { Serum creatinine } \\
(\mathrm{mg} / \mathrm{dl})\end{array}$ & $\begin{array}{c}\text { BUN } \\
(\mathrm{mg} / \mathrm{dl})\end{array}$ & $\begin{array}{c}\text { Urinary albumin } \\
(\mathrm{mg} / \mathrm{dl})\end{array}$ & $\begin{array}{c}\text { eGFR } \\
\left(\mathrm{ml} / \mathrm{min} / 1.73 \mathrm{~m}^{2}\right)\end{array}$ & $\begin{array}{c}\text { UACR } \\
(\mathrm{mg} / \mathrm{g})\end{array}$ \\
\hline Mean reduction & 40.4 & 57.5 & 0.9 & 0.1 & 3.6 & 78.2 & -0.6 & 7.2 \\
\hline
\end{tabular}

Table 2: Adverse events seen in patients

\begin{tabular}{|l|l|c|c|c|}
\hline & Adverse events & No of patients & No of episodes & Percentage of patients \\
\hline 1 & Hypoglycaemia & 18 & 24 & 4.44 \\
\hline 2 & Constipation & 5 & 8 & 1.23 \\
\hline 3 & Gastritis & 3 & 5 & 0.74 \\
\hline & Total & 26 & 37 & 6.41 \\
\hline
\end{tabular}

Among all the patients, $6.41 \%$ of patients experienced adverse event. Hypoglycemia was the most common adverse event seen in $4.44 \%$ of patients followed by constipation $(1.23 \%)$ and gastritis $(0.74 \%)$ (Table 2$)$ 


\section{International Journal of Innovative Research in Medical Science (IJIRMS) Volume 04 Issue 01 Jan 2019, ISSN: 2455-8737, Imp. Factor - 4.102 \\ Available online at - www.ijirms.in}

\section{Discussion}

Teneligliptin and linagliptin are the only DPP4 inhibitors which need no dosage adjustment in renal impairment. ${ }^{[8,9]}$ DPP4 inhibitors have shown antifibrotic and anti-inflammatory activity in kidney disease. DPP4 enzyme is also involved in the advanced glycation end product - receptor axis, which is highly relevant in diabetic kidney disease. ${ }^{[10]}$ The chances of hypoglycaemia are increased in kidney disease due to decreased clearance of insulin and reduced renal gluconeogenesis. ${ }^{[11]}$ Therefore $\mathrm{ADA}^{[12]}$, American Association of Clinical Endocrinologists (AACE) ${ }^{[13]}$ and Kidney Disease Outcomes Quality Initiative (KDOQI $)^{[11]}$ guidelines recommend less stringent HbA1c goals ( $<7 \%$-KDOQI, $<8 \%$-ADA and 7-8\%-AACE) for patients of diabetes with CKD. Published data emphasizes on clinical importance of teneligliptin in management of glycaemic control in diabetic kidney disease. Teneligliptin is not much studied in the Asian Indian diabetic population $^{[10]}$ and even less in those with CKD. Therefore, we planned a study to assess the efficacy and tolerability of teneligliptin in patients of T2DM with CKD.

In the present study, teneligliptin reported significant mean reduction in FPG and PPBG by 40.4 and $57.5 \mathrm{mg} / \mathrm{dl}$ respectively. At 12 weeks, statistically significant $0.9 \%$ reduction in $\mathrm{HbA1c}$ was noted. Effects on renal parameters were also found significantly positive in this study, with reduction of Sr. creatinine by around $4 \%$ and reduction in the BUN of around $9 \%$. There was decrease in mean urinary albumin and UACR and increase in mean eGFR, though it was not statistically significant. Among all the patients, $6.41 \%$ of patients experienced adverse event. Hypoglycemia was the most common adverse event seen in $4.44 \%$ of patients followed by constipation $(1.23 \%)$ and gastritis $(0.74 \%)$. These results are clinically relevant and important in daily practice since renal disease alters the pharmacokinetics of most DPP4 inhibitors. ${ }^{[10]}$

To the best of our knowledge, only one similar study was reported by Shah $\mathrm{K}^{[10]}$ in 2017 in Asian Indian diabetic population to assess the efficacy and safety of teneligliptin in patients of Type 2 Diabetes Mellitus (T2DM) with early Diabetic Kidney Disease (DKD). It was a single centre, retrospective analysis of 37 patients with early DKD, who received teneligliptin $20 \mathrm{mg}$ once daily for 24 weeks. Compared to baseline, the study reported significant reduction in Fasting Plasma Glucose (FPG) (mg/dl) $(143.89 \pm 28.26$ vs. 125.78 $\pm 20.52, \mathrm{p}=0.001)$; Postprandial Glucose (PPG) $(\mathrm{mg} / \mathrm{dl})$ (200.62 \pm 41.88 vs. $165.76 \pm 26.02, \mathrm{p}=0.001)$; Glycated Haemoglobin $(\mathrm{HbA1c})(\%)(8.65 \pm 0.58$ vs. $8.17 \pm 0.54, \mathrm{p}=0.001)$ at 12 weeks. This trend was further continued for 24 weeks with significant reduction in mean FPG, PPG and HbAlc. Significant improvement in serum creatinine $(\mathrm{mg} / \mathrm{dl})(2.45 \pm 0.27$ vs. $2.26 \pm 0.23, \mathrm{p}=0.001)$ and eGFR (CKD-EPI), $\quad\left(\mathrm{ml} / \mathrm{min} / 1.73 \quad \mathrm{~m}^{2}\right) \quad(53.35 \pm 4.24 \quad$ vs. $55.08 \pm 4.19$, $\mathrm{p}=0.001$ ) was noted at 12 weeks and continued for 24 weeks. At baseline, proteinuria was noted in all patients while at 24 weeks, $40.5 \%(\mathrm{p}=0.001)$ patients did not report proteinuria. Except for proteinuria and eGFR, this results were similar to our study.

The efficacy and safety of linagliptin and teneligliptin on glycemic control in T2DM with chronic kidney disease (CKD) was assessed in a pilot study by Tanaka et $\mathrm{al}^{[14]}$ through continuous glucose monitoring. This was a randomized crossover study including 13 patients with T2DM with CKD having HbA1c $<9 \%$ and estimated glomerular filtration rate $<60 \mathrm{~mL} / \mathrm{min} / 1.73 \mathrm{~m}^{2}$. There were two groups receiving either teneligliptin or linagliptin for 6 days from hospital day 5 and followed by crossover to linagliptin or teneligliptin on hospital day 11 for the next 6 days. The primary outcome of changes in the mean amplitude of glucose excursions was similar in both the groups. A similar incidence of hypoglycemia was noted. The study concluded that in patients with T2DM with CKD, teneligliptin and linagliptin have comparable effects on blood glucose and a similar safety profile.

The efficacy and safety of teneligliptin in diabetic ESRD patients undergoing hemodialysis was studied by Otsuki et al ${ }^{[15]}$ through a bicenter, prospective, nonrandomized study in 45 patients for 28week study period, which included 16 patients enrolled in the teneligliptin group and 29 in the control group. At 4 weeks, 36.7 $\mathrm{mg} / \mathrm{dL}$ reduction in blood glucose was noted in the teneligliptin group $(P<0.05)$. More prominent reduction of $3.1 \%$ in glycated albumin was noted in the teneligliptin group than the control group $(P<0.05)$. Similarly, more reduction in HbA1c (difference $-0.57 \% ; P=0.057$ ) was noted in the teneligliptin group compared to the control group. Reduction in these variables was also noted in patients who were switched to teneligliptin from voglibose $0.2 \mathrm{mg}$ three times in a day (tid) or vildagliptin $50 \mathrm{mg}$ once per day (qd). Teneligliptin was well tolerated without any episodes of hypoglycemia during the study period. The study concluded that in diabetic patients with ESRD, teneligliptin significantly improved glycemic control and was well tolerated.

There are few studies reporting efficacy of teneligliptin in reduction of FBS, PPBS and HbA1c in T2DM patients. Agarwal et $\mathrm{al}^{[16]}$ conducted a study in 237 patients with T2DM and inadequate glycemic control and were randomly assigned to receive teneligliptin $20 \mathrm{mg}$ or placebo. Patients of the teneligliptin group showed reduced $\mathrm{HbA} 1 \mathrm{c}$ levels of $-0.304 \%$ after 16 weeks of treatment, Target HbAlc level was achieved by a greater proportion of teneligliptin group patients than placebo group patients. Reduction in FBS and PPBS levels was also higher in teneligliptin group than placebo group which was statistically significant.

\section{Limitations}

This was a 12-week study done in relatively small sample of 405 patients. A multi-centric study with large sample size and longer duration needs to be done for more significant results. Also due to limited resources, we could not evaluate biomarkers of kidney impairment like P-selectin (sP-selectin), Platelet-Derived Microparticles (PDMPs) and Plasminogen Activator Inhibitor 1 (PAI-1) and glycated albumin which are important indicators of kidney disease in diabetes.

\section{Conclusion}

This study, the only prospective study with relatively large sample size in Indian patients of T2DM with CKD, reported significant improvement in glycaemic control, serum creatinine and BUN with teneligliptin. This may have a significant impact in clinical therapeutics for the management of T2DM patients with renal impairment.

\section{Disclosures}

This study was done with TEN 20 brand of Teneligliptin from Centaur Pharmaceuticals Pvt. Ltd. 
Conflict of Interest - None

\section{References}

[1] Ramachandran, Ambady \& Chamukuttan, Snehalatha. (2009). Current scenario of diabetes in India. Journal of diabetes. 1. 18-28.

[2] Wild S, Roglic G, Green A, Sicree R, King H. Global prevalence of diabetes-estimates for the year 2000 and projections for 2030. Diabetes Care. 2004;27 (3):104753.

[3] Kaveeshwar SA, Cornwall J. The current state of diabetes mellitus in India. Australas Med J. 2014;7(1):45-8.

[4] Wu B, Bell K, Stanford A, et al Understanding CKD among patients with T2DM: prevalence, temporal trends, and treatment patterns-NHANES 2007-2012 BMJ Open Diabetes Research and Care 2016;4: e000154.

[5] Singh AK. Efficacy and safety of teneligliptin. Indian J Endocrinol Metab. 2017;21 (1):11-17.

[6] Singh AK. Incretin response in Asian type 2 diabetes: Are Indians different?. Indian J Endocrinol Metab. 2015;19 (1):30-8.

[7] Vella A. Mechanism of action of DPP-4 inhibitors--new insights. J Clin Endocrinol Metab. 2012;97 (8):2626-8.

[8] Sharma SK, Panneerselvam A, Singh KP, Parmar G, Gadge P, Swami OC. Teneligliptin in management of type 2 diabetes mellitus. Diabetes Metab Syndr Obes. 2016; 9:251-60.

[9] Full Prescribing Information: Tradjenta ${ }^{\circledR}$ (linagliptin) tablets [Internet] [accessed on 13th Dec 2018]. Available at: http://www.accessdata.fda.gov/drugsatfda_docs/label/20 15/201280s011lbl.pdf.

[10] Shah K. Teneligliptin in Early Diabetic Kidney Disease: An Observation in Asian Indian Patients with Type 2 Diabetes Mellitus in Real-Life Scenario. J Clin Diagn Res. 2017;11 (6):OC22-OC25.

[11] Diabetes - The National Kidney Foundation. KDOQI Clinical Practice Guideline For Diabetes: Update 2012 [Internet] [cited 2018 Dec 14]. Available from: https://www.kidney.org/professionals/guidelines/guidelin es_commentaries/diabetes-0.

[12] Section 5. Glycaemic Targets. Diabetes Care. 2016;39 (Supplement 1):S39-46.

[13] Type 2 Diabetes Glucose Management Goals. AACE Diabetes Resource Center. [Accessed on 14th Dec 2018]. Available at: http://outpatient.aace.com/type-2diabetes/management[Internet]

[14] Tanaka K, Okada Y, Mori H, et al. Efficacy of linagliptin and teneligliptin for glycemic control in type 2 diabetic patients with chronic kidney disease: assessment by continuous glucose monitoring; a pilot study. Diabetol Int. 2016. pp. 1-7.

[15] Otsuki H, Kosaka T, Nakamura K, Shimomura F, Kuwahara Y, Tsukamoto T. Safety and efficacy of teneligliptin: a novel DPP-4 inhibitor for hemodialysis patients with type 2 diabetes. Int Urol Nephrol. 2014;46 (2):427-432.

[16] Agarwal P, Jindal C, Sapakal V. Efficacy and safety of teneligliptin in Indian patients with inadequately controlled Type 2 diabetes mellitus: A randomized, double-blind study. Indian J Endocr Metab 2018; 22:416. 